Proceedings

\title{
A Tubing-Free, Microfluidic Platform for the Realization of Physiologically Relevant Dosing Curves on Cellular Models ${ }^{+}$
}

\author{
Christian Lohasz ${ }^{1, *}$, Olivier Frey ${ }^{2}$, Kasper Renggli 1,* and Andreas Hierlemann ${ }^{1}$ \\ 1 ETH Zurich, Department of Biosystems Science and Engineering, 4058 Basel, Switzerland; \\ andreas.hierlemann@bsse.ethz.ch \\ 2 InSphero AG, 8952 Schlieren, Switzerland; olivier.frey@insphero.com \\ * Correspondence: christian.lohasz@bsse.ethz.ch (C.L.); kasper.renggli@bsse.ethz.ch (K.R.); \\ Tel.: +41-61-387-3128 (C.L.); +41-61-387-3238 (K.R.) \\ † Presented at the Eurosensors 2017 Conference, Paris, France, 3-6 September 2017.
}

Published: 5 September 2017

\begin{abstract}
We present a microfluidic tilting platform with gravity-driven flow that enables culturing of three-dimensional microtissues under in vivo-like drug dosing regimens. In contrast to traditional, constant dosing regimens, the chip allows for gradual changes in substance concentrations. Dosing gradients are generated through an asymmetric Y-junction of microfluidic channels of different widths. Changes in the chip operation parameters, e.g., different tilting angles, enable to alter the drug dosage on demand. The concentration changes over time matched the predicted values, and preformed microtissues could be cultured in the chip system for at least $24 \mathrm{~h}$. The chip represents an easy-to-handle tool for toxicity and efficacy testing of transient drug concentrations changes.
\end{abstract}

Keywords: microfluidics; tilting chip; pharmacokinetics; 3D microtissues

\section{Introduction}

During the last decades, screening methods for efficacy and toxicity of drugs moved away from animal models towards human in vitro cell culture models [1]. This paradigm change offers the potential to better predict the impact of drugs on the human body when using adequate cell culture models. Especially sophisticated 3D in vitro tissue models, e.g., organotypic multicellular microtissues (MTs), organoids or ex vivo organs, closely reproduce tissue morphology and functionality, and the mechanical properties and biochemical environment of native 3D phenotypes of living organs [2].

In vitro toxicity studies routinely include constant compound dosage over a defined time. These profiles, however, do not represent the in vivo situation. Upon repeated uptake of certain substances, their concentration in the human body gradually increases until a steady state is reached (Figure 1). Simplified protocols often lead to false positive results during toxicity and efficacy testing [3]. To realize physiologically relevant conditions in vitro, multiple pipetting steps or experimental setups with complex pumping schemes are applied to date (Figure 1). 


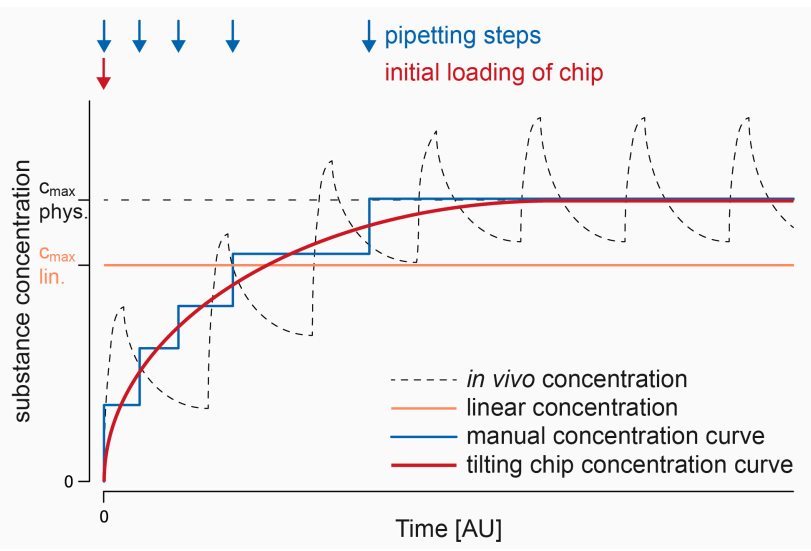

Figure 1. Schematic illustration of different in vivo and in vitro dosing curves. While linear exposure profiles (orange) may lead to false results during efficacy or toxicity testing, manual modulation of the concentration (blue) entails multiple, laborious pipetting steps. The tilting platform enables physiologically relevant, gradual changes of substance concentrations (red) without additional experimental steps.

Recently, we presented a tubing-free microfluidic platform using gravity-driven flow perfusion through platform tilting [4]. The platform allows for robust and parallelized culturing of multiple, interconnected microtissues and for observing their reactions upon exposure to compounds. Here, we modified our existing chip system to enable variable dosage protocols. The chip features three liquid reservoirs, two of which are located on the same side. Both are connected to the main channel trough differently sized channels (Figure 2a). Upon filling the two reservoirs on the left side with cell culture medium (wide channel) and a treatment solution (narrow channel), the liquids combine in the asymmetric Y-junction and then pass a mixing structure before reaching the MTs. Barrier structures in the spheroid compartments partially surround the introduced MTs (Figure 2b,c) to protect them against direct exposure to medium flow and resulting shear stress. A computational fluid dynamics simulation (COMSOL Multiphysics, Figure 2d) was used to show this effect. With every tilting cycle, more of the treatment substance is released into the system so that the concentration gradually increases (Figure 3a), until an equilibrium in the main channel is reached.

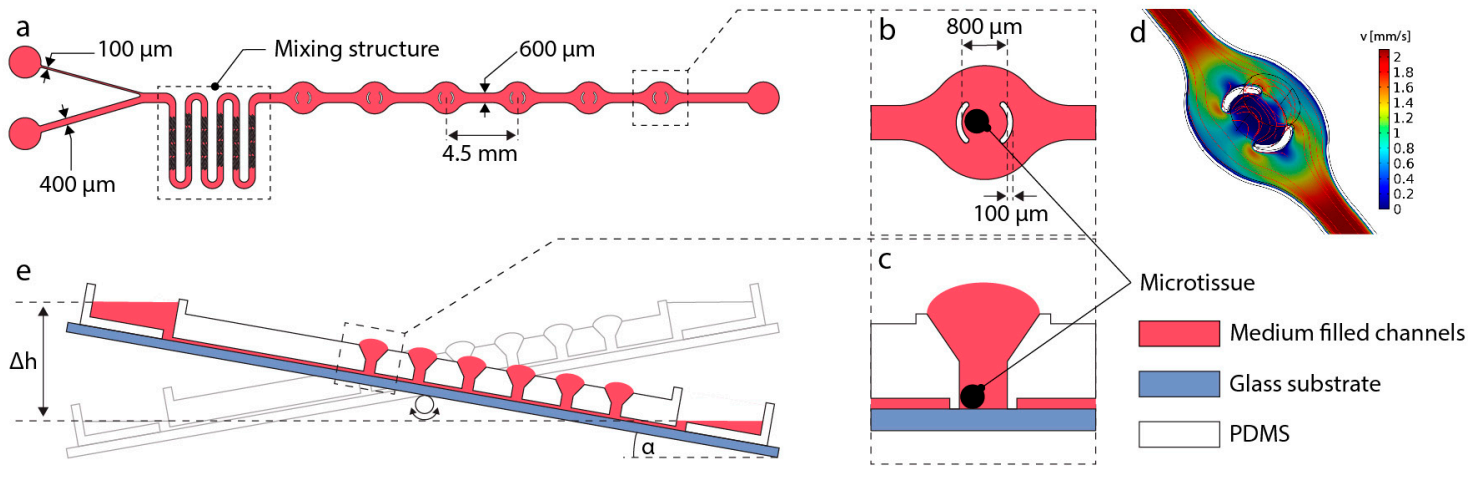

Figure 2. Design and operation of the microfluidic tilting platform (a) Channel layout of the device with a Y-junction to produce physiologically relevant dosing. The channel harbors a mixing structure as well as spheroid compartments. (b) Close-up top view and (c) side view of a spheroid compartment, depicting the standing drop on top of the compartment for optimal loading and the barrier structures protecting the spheroid from flow-dependent shear stress. (d) Assessment of the liquid velocity around the microtissue compartment to estimate shear stress (flow rate of $10 \mu \mathrm{L} \mathrm{min}^{-1}$ ). (e) Tilting of the platform by an angle $\alpha$ induces gravity-driven perfusion of the channels according to the height difference $\Delta \mathrm{h}$. 
a

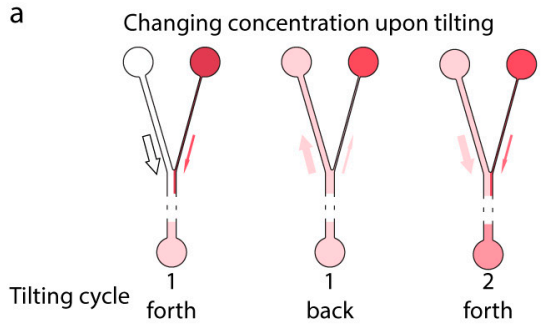

$\mathrm{b}$

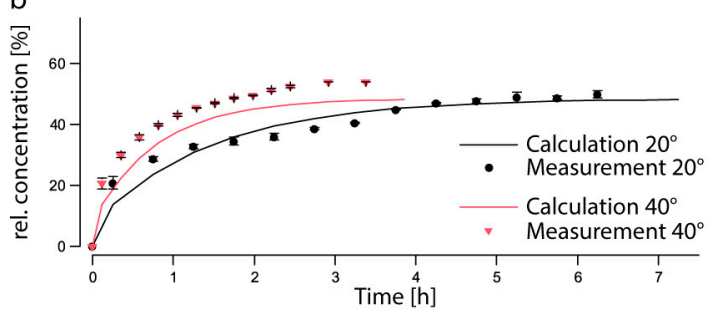

Figure 3: Changing concentrations upon tilting. (a) Schematic depicting the unequal flow through the differently sized channels (indicated by arrow thickness) and the changing concentrations within the three reservoirs. (b) Experimental assessment of the concentration changes in the main channel and reservoir using amaranth red in the dosing reservoir at tilting angles of $20^{\circ}$ and $40^{\circ}$. Measured values generally match the calculations ( $n=3$ measurements; data represent average values $\pm S D$ ).

\section{Methods}

\subsection{Fabrication}

The device was fabricated in PDMS using a SU-8 bottom mold and a PDMS top mold. For the SU-8 mold, previously reported microfabrication processes with two layers were used [4]. The first $100-\mu \mathrm{m}$-thick layer includes the channel structures and MT compartments with their barriers. The herringbone structures for efficient mixing of the two liquids ( $25 \mu \mathrm{m}$ in height) were designed as recommended by Stroock, et al. [5].

The top mold includes all surface structures (reservoirs and MT loading ports). A 3D-printed template (Accura 3350, Proto Labs, Germany) was used to cast the PDMS top mold. Treatment with trichlorosilane prevented the fusion of the top mold with the final chip and enabled multiple usage of the mold.

The casted PDMS chips were $\mathrm{O}_{2}$-plasma-bonded to standard microscopic glass slides to close the channels. All channels, reservoirs and MT compartments were then coated with Biolipidure 206 to render them hydrophilic and to prevent MTs from attaching to the surfaces.

\subsection{Device Operation}

Channels and reservoirs were initially flooded with cell culture medium. Next, preformed spherical MTs were harvested from a microplate with a pipette and transferred to their designated compartments.

In vivo-like treatment was initiated by filling the reservoirs on the side of the Y-junction with plain medium (wide channel) and medium containing a substance of interest (narrow channel). Repetitive tilting by an angle $\alpha$ induced a controlled concentration increase of the substance in the main channel and the MT compartments. The concentration changes by the unequal volumes of liquid flowing from the two reservoirs into the common channel during one tilting interval. Upon flowing backwards to the Y-junction, the liquid is assumed to split up at the same ratio at which it entered the main channel (Figure 3a). By changing the tilting angle $\alpha$, the dosing can be modulated. At larger angles, a higher flow rate is achieved, which results in faster mixing of the two solutions and a steeper concentration curve. The opposite holds true for smaller angles with lower flow rates and comparably flat curves.

The entire experimental setup including a GravityFLOW ${ }^{\mathrm{TM}}$ tilting device (InSphero AG, Switzerland) and a humidity chamber, harboring the chips, can be operated in a cell culture incubator at standard mammalian cell culture conditions $\left(37^{\circ} \mathrm{C}, 5 \% \mathrm{CO}_{2}, 95 \%\right.$ humidity). Stackable humidity chambers hold up to 4 chips, which enables higher throughput and parallelized operation. 


\subsection{Assessment of Concentration Changes}

Based on the channel dimensions, their hydrostatic resistances and the resulting flow rates the tilting-dependent changes of concentrations in the main channel were calculated. The changes were tracked using amaranth red in $\mathrm{H}_{2} \mathrm{O}$ in the dosing reservoir with the narrow channel and plain $\mathrm{H}_{2} \mathrm{O}$ in the wide channel. After every tilting cycle, the absorbance of the liquid in the shared reservoir was measured at a wavelength of $520 \mathrm{~nm}$ in reference to the loading solution using a NanoDrop2000 (ThermoScientific, Switzerland).

\subsection{Microtissue Analyses}

To track changes of the MTs upon substance exposure, morphology and size, as well as secreted albumin and intracellular ATP were measured.

\section{Results and Discussion}

For concentration measurements over time, tilting angles of $20^{\circ}$ and $40^{\circ}$ were chosen to demonstrate the modulation of the dosing curve. The measurements evidenced a good correlation to calculated values for both tilting angles and demonstrated that gradual concentration changes could be predicted and achieved (Figure $3 b$ ). The modulation of the tilting angle changed the slope of the dosing curve, allowing to mimic curves that resemble the pharmacokinetics of common substances.

We also assessed the performance of previously formed human liver microtissues (hLiMT, InSphero AG, Switzerland) in the device. The MTs were cultured for $24 \mathrm{~h}$ in human liver maintenance medium (hLiMM, InSphero AG, Switzerland) and compared to static well-plate-cultured spheroids using standard mammalian cell culture conditions. Images of the microtissues (Figure 4c) did not show significant differences between static and perfused culturing conditions and confirmed that the spheroids preserve their compact morphology even under flow and light shear stress. Assessment of microtissue viability (Figure 4a) according to intracellular ATP concentrations (CellTiterGlo 3D Cell Viability Assay, Promega, Switzerland) showed similar average values for culturing that was performed under perfusion and static control conditions. Detection of secreted albumin (Albumin ELISA Kit, Bethyl Laboratories, Inc., Montgomery, TX, USA; Figure 4b), a liver specific marker, in the supernatant of both conditions showed higher secretion rates for hLiMT cultured under perfusion on chip. The increased microtissue functionality, shown by albumin secretion, in combination with their similar viability demonstrate the advantages of using perfused in vitro systems in comparison to standard static cell culture conditions.
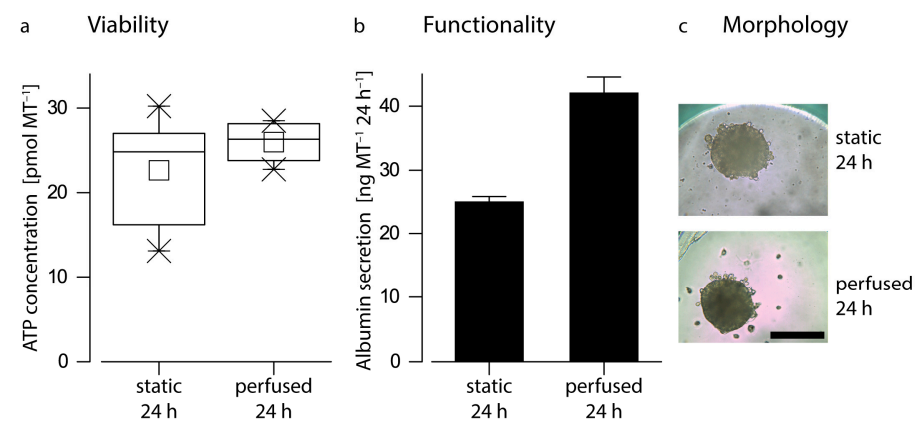

Figure 4. Testing of the biocompatibility of the chip by assessment of (a) microtissue viability by measuring intracellular ATP, (b) functionality by quantification of secreted albumin and (c) monitoring of the microtissue morphology. Human liver microtissues were equally viable, similar in morphology and featured increased functionality in comparison to control samples cultured under static conditions ( $n=4-6$ MTs; scale bar: $250 \mu \mathrm{m}$ )

\section{Conclusions and Outlook}

In this paper, an easy-to-use in vitro method for the exposure of 3D microtissues to continuously varying concentrations of a substance of interest was presented. By using the proposed channel 
structures on a tilting device, gradually increasing concentrations to mimic physiological, in vivolike concentration changes upon drug dosing could be achieved without the need for time-consuming pipetting schemes or sophisticated pumping protocols.

We showed the culturing of a sensitive cell type, hLiMTs, in the device, which demonstrates the potential of the device for many other 3D cell culture models. The combination of different, organspecific microtissues and their interplay can be monitored in the presented setup, e.g., by combining liver and cancer microtissues so that efficacy and toxicity of cancer treatments could be simultaneously evaluated.

Acknowledgments: This work was financially supported by CTI grant 18024.1 PFLS-LS.

Author Contributions: C.L., O.F., K.R. and A.H. conceived and designed the experiments; C.L. performed the experiments; C.L. and O.F. analyzed the data; C.L., K.R. and A.H. wrote the paper.

Conflicts of Interest: The authors declare no conflict of interest.

\section{References}

1. Leist, M.; Hartung, T.; Nicotera, P. The dawning of a new age of toxicology. ALTEX 2008, 25, 103-114, doi:10.14573/altex.2008.2.103.

2. Griffith, L.G.; Swartz, M.A. Capturing complex 3D tissue physiology in vitro. Nat. Rev. Mol. Cell Biol. 2006, 7, 211-224, doi:10.1038/nrm1858.

3. Tsaioun, K.; Blaauboerand, B.J.; Hartung, T. Evidence-based absorption, distribution, metabolism, excretion (ADME) and its interplay with alternative toxicity methods. ALTEX 2016, 33, 343-358, doi:10.14573/altex.1610101.

4. Kim, J.Y.; Fluri, D.A.; Marchan, R.; Boonen, K.; Mohanty, S.; Singh, P.; Hammad, S.; Landuyt, B.; Hengstler, J.G.; Kelm, J.M.; et al. 3D spherical microtissues and microfluidic technology for multi-tissue experiments and analysis. J. Biotechnol. 2015, 205, 24-35, doi:10.1016/j.jbiotec.2015.01.003.

5. Stroock, A.D.; Dertinger, S.K.; Ajdari, A.; Mezic, I.; Stone, H.A.; Whitesides, G.M. Chaotic mixer for microchannels. Science 2002, 295, 647-651, doi:10.1126/science.1066238.

(C) 2017 by the authors. Licensee MDPI, Basel, Switzerland. This article is an open access article distributed under the terms and conditions of the Creative Commons Attribution (CC BY) license (http://creativecommons.org/licenses/by/4.0/). 